\title{
Regional Differences in Microtubule Dynamics in the Axon
}

\author{
Fridoon J. Ahmad, Thomas P. Pienkowski, and Peter W. Baas \\ Department of Anatomy, The University of Wisconsin Medical School, Madison, Wisconsin 53706
}

We have used an Indirect method to compare the dynamic properties of microtubules (MTs) in the main shaft and distal regions of the axon. Individual MTs are staggered along the length of the axon and consist of a labile domain situated at the plus end of a stable domain (Baas and Black, 1990). As a result of this organization and the plus-end-distal orientation of axonal MTs, the most distal region of the axon consists entirely of labile domains, while the main shaft consists of a mixture of labile and stable domains. In this study, we wished to determine whether the labile domains extending into the distal axon differ in their dynamic properties from the labile domains terminating in the main shaft. To address this issue, we used immunoelectron microscopy to compare the tyrosination state of the labile domains terminating in these 2 axon regions. Because detyrosination is a polymerspecific modification of $\alpha$-tubulin that accumulates with time, the levels of tyrosinated $\alpha$-tubulin will be a reflection of the age, and hence dynamic properties, of the polymer. To maximize our chances of visualizing potential differences, we varied the concentration of the primary antibody in these experiments. Our studies indicate that the stable domains are generally deficient in tyrosinated $\alpha$-tubulin, while the labile domains contain clearly detectable levels. Within the labile domain, the subsection closer to the plus end of the MT contains relatively higher levels of tyrosinated $\alpha$-tubulin than does the subsection farther from the plus end, suggesting that the levels of tyrosinated $\alpha$-tubulin in the labile domain may gradually increase as one moves away from the stable domain toward the plus end of the MT. Although these observations apply to the labile domains in both regions of the axon, the labile domains extending into the distal region contain comparatively higher levels of tyrosinated $\alpha$-tubulin than do the labile domains terminating in the main shaft. These results are consistent with the view that highly dynamic MT polymer is present throughout the axon, but that the polymer nearest the advancing growth cone is particularly dynamic.

\footnotetext{
Received June 18, 1992; revised Aug. 25, 1992; accepted Sept. 1, 1992.

We thank Kelly Cimbalnik, Janet Christenson, Theresa Wong, and Dan Uhlrich of The University of Wisconsin-Madison for expert assistance. We also thank Mark Black and Anthony Brown of Temple University for helpful advice, Mark Black for providing NGF for our cultures, Janet Krotzman and the blood bank at St. Mary's Hospital in Madison, WI, for providing human placental serum for our cultures, Gianni Piperno of The Mount Sinai Medical Center for providing 6-11b-1 antibody, and The National Cancer Institute for providing taxol, a necessary component of our microtubule stabilizing buffer. This work was funded by National Institutes of Health Grant NS 28785 to P.W.B. In addition, P.W.B. is the recipient of a Research Career Development award from the National Institutes of Health.

Correspondence should be addressed to Dr. Peter W. Baas, Department of Anatomy, The University of Wisconsin Medical School, 1300 University Avenue, Madison, WI 53706.

Copyright (C) 1993 Society for Neuroscience $0270-6474 / 93 / 130856 \sim 11 \$ 05.00 / 0$
}

[Key words: microtubule, axon, growth cone, tyrosinated $\alpha$-tubulin, microtubule stability, microtubule dynamics, immunoelectron microscopy]

Microtubules (MTs) are dynamic structural elements that are essential for the growth and maintenance of the axon. Because of the importance of MTs in defining the architecture and cytoplasmic composition of the axon (for review, see Lasek, 1988; Meininger and Binet, 1989; Black and Baas, 1989), there is great interest in elucidating the mechanisms by which the axonal MT array is elaborated during axon growth. Several recent studies indicate that MT nucleation, assembly, disassembly, rescue, stabilization, bundling, and movement are all important events in the axon (see, e.g., Baas and Black, 1990; Reinsch et al., 1991; Sabry et al., 1991; Tananka and Kirschner, 1991; Baas and Ahmad, 1992). The term MT dynamics has been used to describe the behavior of individual MTs in terms of their alternate bouts of assembly and disassembly, with more dynamic MTs turning over faster than less dynamic MTs (for reviews, see Dustin, 1984; Kirschner and Mitchison, 1986). Although much attention has been given to MT dynamics in the axon, there is considerable controversy concerning the major sites in the axon where MT dynamics occur, and how regional differences in MT dynamics may be important to the growth of the axon.

In recent years, there has been a strong bias that the most distal region of the axon bchind the advancing growth conc is a unique and highly specialized site for MT dynamics in the axon, and that, by comparison, the main shaft of the axon is relatively quiescent. This idea was originally derived from studies showing that axon growth is more sensitive to anti-MT drugs when applied specifically to the growth cone compared to other locations along the axon (Bamburg et al., 1986). More recent photobleach analyses suggest that the MT polymer extending into the growth cone turns over subunits more rapidly than does the polymer in the main shaft of the axon (Lim et al., 1989, 1990). In addition, immunofluorescence analyses indicate that the acetylated variant of $\alpha$-tubulin, which is generally enriched in less dynamic MT polymer, is present at significantly lower levels in the distal axon compared to the main shaft (Robson and Burgoyne, 1988; Lim et al., 1989; Baas and Black, 1990). Although these observations appear to support the idea that the MT polymer extending into the distal axon is more dynamic than the polymer in the main shaft, it is pertinent that both photobleaching and immunofluorescence are unable to resolve the behavior or composition of individual MTs within the dense bundle of MTs in the axon. In light of work indicating that individual MTs in a population can behave very differently from one another (for review, see Kirschner and Mitchison, 1986), it seems possible that higher-resolution techniques could reveal new information not apparent from these low-resolution techniques. 


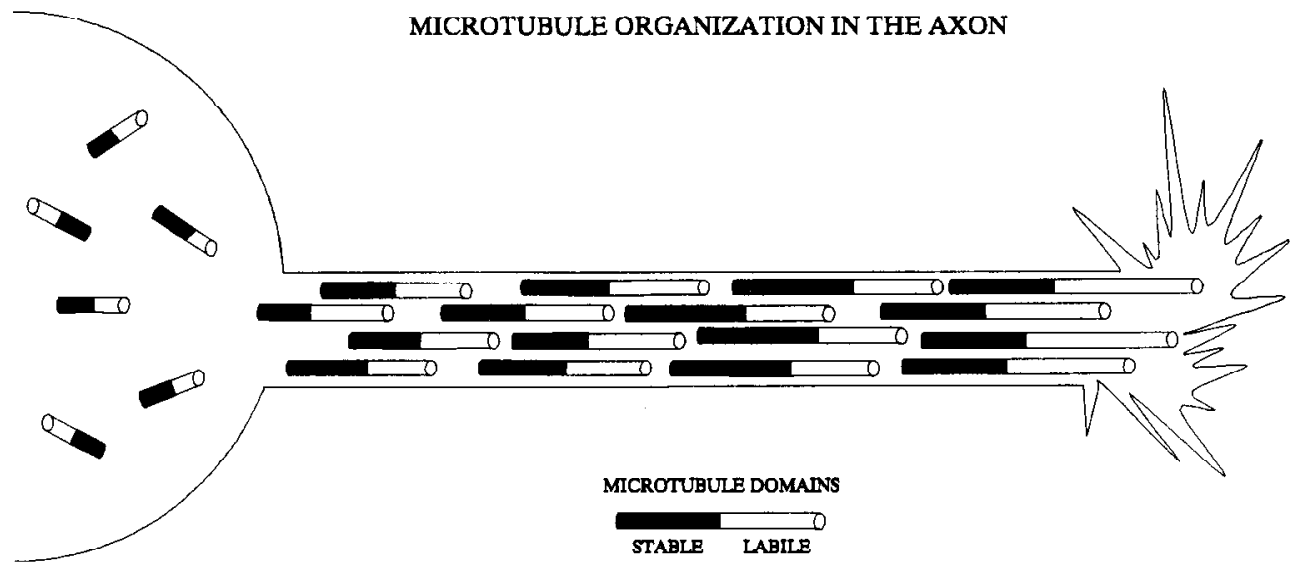

Figure 1. Schematic illustrating the organization of axonal MTs based on information derived from electron microscopic analyses. The plus ends of the MTs are directed away from the cell body. The MTs consist of two domains, stable and labile, with the labile domain situated at the plus end of the stabile domain. Both stable and labile domains are present all along the length of the axon, except in the distal region of the axon, into which extend only the labile domains of MTs originating in the main shaft of the axon. Techniques of insufficient resolution to resolve individual MTs will average the properties of stable and labile polymer in the main shaft, and hence may give the mistaken impression that labile polymer is present only in the distal axon.
Information derived from electron microscopic analyses supports this view, and presents a more complex picture of MT organization and dynamics in the axon. It is now well established that axonal MTs have a uniform polarity orientation, with their plus ends distal to the cell body (Heidemann et al., 1981; Baas et al., 1987, 1988, 1989, 1991), and that axonal MTs stop and start at multiple sites along the length of the axon (Bray and Bunge, 1981; Tsukita and Ishikawa, 1981). In addition, we have recently reported that the axons of cultured sympathetic neurons contain two classes of MT polymer, stable and labile, that differ in their sensitivity to nocodazole by roughly 35 -fold (Baas and Black, 1990; Baas et al., 1991). The labile polymer is more dynamic and is rich in tyrosinated $\alpha$-tubulin, while the stable polymer is generally deficient in this $\alpha$-tubulin variant. Using immunoelectron microscopy to distinguish stable and labile polymers visually based on their content of tyrosinated $\alpha$-tubulin, we determined that these two types of polymer exist in the form of distinct domains on individual MTs in the axon, with the labile domain situated at the plus end of the stable domain. Becausc of the plus-cnd-distal orientation of axonal MTs, the distal region of the axon contiguous with the growth cone contains only labile domains. In contrast, the labile domains of MTs terminating in the main shaft are staggered throughout the axon, and are situated alongside stable domains of other MTs. Because of this organization (depicted schematically in Fig. 1), any technique of insufficient resolution to resolve individual MTs will average the properties of stable and labile domains in the main shaft, and hence fail to provide an accurate comparison of the dynamics of individual MTs terminating in the main shaft and distal regions of the axon.

In the present study, we have sought to compare the dynamic properties of the labile domains of MTs terminating in the main shaft with those extending into the distal region of the axon. As in our previous work, we have focused on tyrosination state as an indirect indicator of MT dynamics, and have taken advantage of the high degree of resolution afforded by immunoelectron microscopy. Detyrosination is a polymer-specific modification of $\alpha$-tubulin that accumulates with time (Gunderson et al., 1987; Bulinski et al., 1988; for more discussion, see Baas et al., 1991). As a result, the levels of tyrosinated $\alpha$-tubulin will be a reflection of the age, and hence dynamic properties, of the polymer. We have extended our previous studies by directly comparing and quantifying the levels of this $\alpha$-tubulin variant in each of the two axon regions using different antibody concentrations. Our goal was to maximize the possibility of visualizing differ- ences, if they exist, between the labile domains of MTs terminating in each of these two axon regions.

\section{Materials and Methods}

Cell culture. Sympathetic neurons from the superior cervical ganglia of newborn rat pups were cultured in two ways. Dissociated cultures were used for immunofluorescence analyses because they permit visualization along substantial lengths of individual axons. Explant cultures were used for electron microscopic analyses because they maximize the number of aligned axons that can be sectioned simultaneously. Methods for the preparation of explant cultures were as previously described (Baas and Black, 1990; Baas and Ahmad, 1992). For improved optical quality, dissociated cultures were grown on polylysine-treated glass coverslips adhered to plastic dishes prepared as previously described (Whitlon and Baas, 1992). In addition, laminin was added to the culture medium to reduce fasciculation of neighboring axons (Higgins et al., 1991). The concentrations of polylysine and laminin were $1 \mu \mathrm{g} / \mathrm{ml}$ and $10 \mu \mathrm{g} / \mathrm{ml}$, respectively, and both were purchased from Sigma Chemical Company (St. Louis, MO).

Immunofluorescence microscopy. For double-label immunofluorescence microscopy, we used a modification of the procedure described by Brown et al. (in press). Dissociated cultures were rinsed once briefly in an MT stabilizing buffer, PHEM [60 mM piperazine- $N, N^{\prime}$-bis[2-ethanesulfonic acid (PIPES), $25 \mathrm{~mm}$ HEPES, $10 \mathrm{~mm}$ EGTA, $2 \mathrm{~mm} \mathrm{MgCl}$, pH 6.9], and then extracted for $5 \mathrm{~min}$ in PHEM supplemented with 10 $\mu \mathrm{M}$ taxol and $1 \%$ saponin. Following extraction, cultures were fixed by the addition of an equal quantity of PHEM containing 4\% paraformaldehyde and $0.1 \%$ glutaraldehyde. After $10 \mathrm{~min}$, cultures were pos$\mathrm{t}$-extracted in $0.1 \%$ Triton $\mathrm{X}-100$ for $15 \mathrm{~min}$, and then, to reduce autofluorescence, treated 3 times for $5 \mathrm{~min}$ each in $10 \mathrm{mg} / \mathrm{ml}$ sodium borohydride. Cultures were then incubated for $30 \mathrm{~min}$ in a blocking solution containing $1 \%$ bovine serum albumin and $2 \%$ normal goat serum, incubated overnight at $4^{\circ} \mathrm{C}$ with primary antibodies diluted in blocking solution, rinsed 3 times for 5 min each with PBS, incubated in blocking solution again for $30 \mathrm{~min}$, incubated for $\mathrm{I} \mathrm{hr}$ at $37^{\circ} \mathrm{C}$ with second antibodies, rinsed 4 times for 5 min each with PBS, and mounted in a medium containing $50 \%$ glycerol and $10 \mathrm{~mm} N$-propyl gallate. The primary antibodies were YL $1 / 2$ (ascites purchased from Accurate Chemical and Scientific Corp., Westbury, NY), a rat monoclonal antibody that recognizes the tyrosinated but not the detyrosinated form of $\alpha$-tubulin (Kilmartin et al., 1982; Wheland et al., 1983), and 6-1 l b1 , a mouse monoclonal that recognizes the acetylated but not the unacetylated form of $\alpha$-tubulin (culture supernatant; see Piperno and Fuller, 1985). These antibodies were used at concentrations of 1:100 and 1:1 respectively. The YL 1/2 was visualized with a fluorescein-conjugated goat anti-rat second antibody, and the $6-11 \mathrm{~b}-1$ was visualized with a Texas red-conjugated goat anti-mouse second antibody. Both of these second antibodies were purchased from 'Accurate, and used at 1:100.

Immunoelectron microscopy. Single-label immunoelectron microscopy was performed on explant cultures as previously described (Baas and Black, 1990; Baas et al., 1991; Baas and Ahmad, 1992). Prior to extraction, the distal regions of axons were circled on the bottom of the culture dish with a diamond-marker objective, and photographed and 

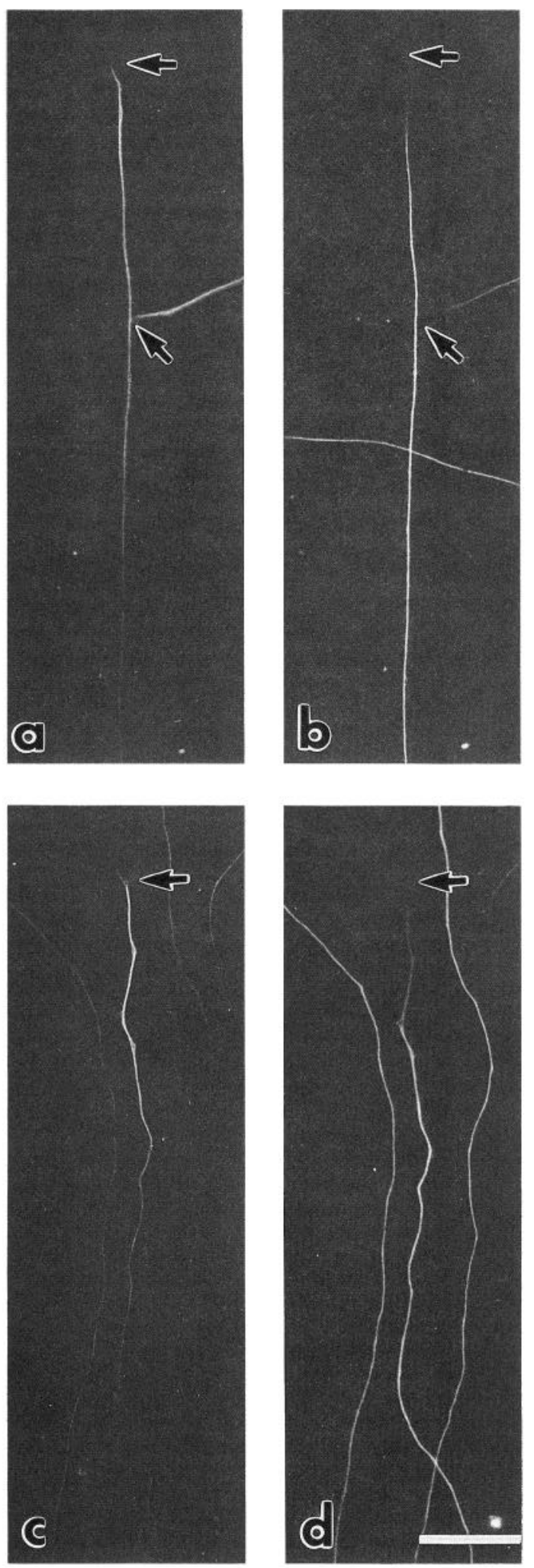

Figure 2. Immunofluorescence analyses on the distribution of tyrosinated and acetylated $\alpha$-tubulin in MT polymer in the main shaft and distal regions of the axon. Cultures were extracted in the presence of an MT stabilizing buffer, and double-labeled with antibodies against these two $\alpha$-tubulin variants as described in Materials and Methods. $a$ and $b$ show the same axon regions, as do $c$ and $d . a$ and $c$ are stained for tyrosinated $\alpha$-tubulin, while $b$ and $d$ are stained for acetylated $\alpha$-tubulin. The entire length of the axon stains for tyrosinated $\alpha$-tubulin, but the most distal region $(\approx 50 \mu \mathrm{m}$ on average) near and including the growth or sketched. The photographs or sketches were subsequently used for the relocation of the distal axon regions on thin sections viewed with the electron microscope. Identification of the termination of individual axons was further refined by using as a marker the neurofilament array, which typically ends a few microns proximal to the distal termination of the MT array. This approach was particularly useful in experiments in which, prior to preparation for immunoelectron microscopy, cultures were treated with $2 \mu \mathrm{g} / \mathrm{ml}$ nocodazole (Aldrich Chemical Co., Milwaukee, WI) for 3 min to depolymerize the most distal several microns at the plus ends of the MTs. After circling and photography, drugged and undrugged cultures were rinsed briefly in PHEM, and then extracted for $10 \mathrm{~min}$ in PHEM supplemented with $10 \mu \mathrm{M}$ taxol, $1 \%$ Triton X-100, and $0.2 \mathrm{M} \mathrm{NaCl}$. The cultures were then fixed by the addition of an equal quantity of PHEM containing $1 \%$ glutaraldehyde. After $10 \mathrm{~min}$ of fixation, cultures were rinsed in PHEM, treated with $2 \mathrm{mg} / \mathrm{ml}$ sodium borohydride in PHEM for $15 \mathrm{~min}$, rinsed in TBS-1 (10 mM Tris, 140 $\mathrm{mm} \mathrm{NaCl}, \mathrm{pH} 7.6$ ), blocked in TBS- 1 containing $5 \%$ normal goat serum, incubated overnight at $4{ }^{\circ} \mathrm{C}$ with the YL $1 / 2$ primary antibody, rinsed extensively with TBS-2 ( $20 \mathrm{~mm}$ Tris, $140 \mathrm{~mm} \mathrm{NaCl}, \mathrm{pH} 8.2)$ containing $0.1 \% \mathrm{BSA}$, incubated for $3 \mathrm{hr}$ with $5 \mathrm{~nm}$ gold-conjugated goat anti-rat second antibody (Amersham Corp., Arlington Heights, IL), rinsed extensively with TBS-2, and then fixed again, osmicated, dehydrated, and embedded for electron microscopy by conventional means. The concentration of the YL 1/2 antibody was varied in different experiments, and was most frequently used at either $1: 200$ or $1: 200,000$. The goldconjugated second antibody was used at 1:2.

\section{Results}

Distribution of $\alpha$-tubulin variants in the axon. In an initial set of studies, double-label immunofluorescence microscopy was used to explore the general distribution of tyrosinated ànd acetylated $\alpha$-tubulin variants in the main shaft and distal regions of the axon. Acetylation, like detyrosination, is a polymer-specific modification of $\alpha$-tubulin that accumulates with the age of the polymer (Black et al., 1989). Our results were generally consistent with those obtained in previous studies of this kind performed on the axons of various types of cultured neurons (Robson and Burgoyne, 1988; Lim et al., 1989; Baas and Black, 1990; Baas et al., 1991). Nevertheless, to interpret better the results of our higher-resolution immunoelectron microscopic work, we wished first to analyze the results of these immunofluorescence experiments in more detail and with greater rigor than has been done in the past. We analyzed 50 different axons, performed measurements of the distances over which each antibody stained with greater or lesser intensity, and generated means and SDs for these distances. As previously reported, both antibodies stain the dense bundle of MTs in the main shaft of the axon relatively uniformly along its length. The overall intensity of staining in the main shaft was dimmer than in our previous studies, presumably because axons grown in the presence of laminin are thinner and not fasciculated with neighboring axons (Higgins et al., 1991), compared to the thicker, fasciculated axons of cultures grown on collagen (Baas and Black, 1990; Baas et al., 1991; Baas and Ahmad, 1992). In the distal region of the axon contiguous with the growth cone, staining for both tyrosinated and acetylated tubulin differ dramatically from the staining in the main shaft. In this region, staining for tyrosinated $\alpha$-tubulin becomes markedly more intense (Fig. $2 a, c$; see also Brown et al., in press),

$\leftarrow$

cone stains more intensely than does the main shaft, in which staining is weak. Staining for acetylated $\alpha$-tubulin is relatively uniform along the main shaft, but drops off to very low or undetectable levels in the most distal $2-10 \mu \mathrm{m}$ of the axon. Virtually all of the axons showed this staining pattern, but the lengths of intense staining for tyrosinated tubulin and deficiency of staining for acetylated tubulin in the distal region varied somewhat from axon to axon (see Results). Arrows mark distal tips of axons. Scale bar, $20 \mu \mathrm{m}$. 
while staining for acetylated $\alpha$-tubulin becomes markedly less intense, often dropping off completely (Fig. $2 b, d$ ). Clearly, however, these two regions of more intense staining for tyrosinated $\alpha$-tubulin and less intense staining for acetylated $\alpha$-tubulin are not entirely overlapping. The former spans the most distal 25$75 \mu \mathrm{m}$ (mean $\pm \mathrm{SD}, 50 \pm 12 \mu \mathrm{m}$ ) of the axon, with the staining gradually decreasing over the next $10-30 \mu \mathrm{m}(20 \pm 14 \mu \mathrm{m})$, reaching a relatively constant level of staining over the remaining portion of the axon. The latter spans the most distal 2-10 $\mu \mathrm{m}(7 \pm 4 \mu \mathrm{m})$, with the staining increasing much more abruptly to reach a relatively constant level over the remaining portion of the axon.

The higher intensity of staining for tyrosinated MT polymer that we observed in the distal region of the axon is consistent with current knowledge indicating that this region is enriched in the labile domains of MTs originating more proximally in the axon, whereas the main shaft contains a mixture of stable and labile domains (see Fig. 1). Moreover, a length of roughly $50 \mu \mathrm{m}$ for this region is predicted by previous data showing that MTs in the axons of cultured neurons have an average length of $\approx 100 \mu \mathrm{m}$ (Bray and Bunge, 1981), combined with our observation that roughly half the total polymer in the axon is labile (Baas and Black, 1990; Baas et al., 1991). In addition, the gradual decrease in staining moving from the distal axon into the main shaft is consistent with the staggered, nonoverlapping organization of the stable and labile domains of neighboring MTs (see Fig. 1), but may also be indicative of a gradient of detyrosination along the length of individual MTs, such as has been observed in the case of trypanosome MTs (Sherwin and Gull, 1989). The fact that only the most distal $2-10 \mu \mathrm{m}$ of the axon show a significant drop in staining for acetylated $\alpha$-tubulin indicates that staining for acetylated and tyrosinated $\alpha$-tubulins provide different types of information about MT dynamics in the distal region of the axon. While staining for tyrosinated $\alpha$-tubulin is a marker for the entire labile domain of the MT, the lack of staining for acetylated $\alpha$-tubulin apparently serves as a marker for only a fraction of the labile domain, that region closest to the plus end of the MT. In previous studies at the immunoelectron microscopic level, attempts were made to distinguish better the labile polymer from stable polymer by decreasing the concentration of the antibody against acetylated $\alpha$-tubulin (Baas et al., 1991). However, this resulted in patchy staining that was difficult to quantify. For these reasons, we have focused our immunoelectron microscopic efforts specifically on tyrosinated $\alpha$-tubulin, the more useful marker.

Immunoelectron microscopic analyses of MT tyrosination state in different regions of the axon. To compare the levels of tyrosinated $\alpha$-tubulin in the labile domains of MTs terminating in the main shaft and distal regions of the axon, we took advantage of the high degree of resolution afforded by immunoelectron microscopy. We previously reported that the labile MT polymer of the axon could be visually distinguished from the stable polymer by the specific staining of the former for tyrosinated $\alpha$-tubulin (Baas and Black, 1990; Baas et al., 1991). In these previous studies, we used the YL 1/2 antibody at a concentration of 1:200 and scored all MT polymer as either stained or unstained (although we observed some variation in the density of gold particles on the stained polymer). In this study, we repeated the immunoelectron microscopic analyses using different concentrations of the YL $1 / 2$ antibody, and quantified the proportion of stained polymer and the density of gold particles on the stained polymer in each region of the axon. If the polymer extending into the distal region of the axon is more dynamic than the polymer terminating in the main shaft of the axon, we would expect to find staining conditions that would reveal higher levels of tyrosinated $\alpha$-tubulin in the distal polymer.

Because of the possibility that there may be a gradient in $\alpha$-tubulin composition along individual labile domains, we separately analyzed two subsections along their length. The first subsection comprised the most distal 2-10 $\mu \mathrm{m}$ nearest the plus end of the MT (subsection I), and the second comprised a distance $\approx 20-40 \mu \mathrm{m}$ from the plus end (subsection II). Because of the potential for confusion, we stress that this new terminology is used to denote parts of an individual $M T$, and not parts of the axon itself. In the distal region of the axon, the subsections of the labile domain can be distinguished from one another with direct measurements along the axon hecause all of the MTs terminate comparatively synchronously (within a few microns of one another), as do the growth cones themselves in certain advantageous regions of the explant cultures (Fig. 3). In the main shaft, direct measurements along the lengths of individual MTs were impossible due to their staggered arrangement along the axon's length (see Fig. 1). Thus, for the main shaft, one cannot directly discern polymer that comprises each subsection of the labile domains of the MTs. For this reason, it was necessary to use an experimental approach to dissect apart the two subsections of the labile domains. We reasoned that, because MT dynamics occur only from the plus ends of MTs in the axon (Baas and Ahmad, 1992; see also Okabe and Hirokawa, 1988), the most distal few microns of the MTs could be selectively depolymerized using a bricf nocodazole treatment. According to our previous quantitative work on the loss of MT mass in the presence of nocodazole, $3 \mathrm{~min}$ of drug treatment at $2 \mu \mathrm{g} / \mathrm{ml}$ is sufficient to shorten the labile domains by $\approx 10-20 \mu \mathrm{m}$ (Baas and Black, 1990; Bass et al., 1991), thus removing subsection I of the labile domain while leaving subsection II intact. This is because subsection I is situated at the plus end of the MT, and therefore must be depolymerized before subsection II could be depolymerized. [We note that the kinetics of drug-induced depolymerization suggest that subsection II is no more stable than subsection I (Baas and Black, 1990; Baas et al., 1991). Thus, current information suggests that subsection II takes more time to depolymerize simply because it must first await for subsection II to depolymerize, and not because of stability differences along the length of the labile domain.] If there is a gradient of tyrosination along the length of the labile domain in either or both axon regions, we would expect to find higher levels of tyrosinated $\alpha$-tubulin in subsection I compared to subsection II in one or both of the axon regions. (Fig. 7, presented in the Discussion, schematically summarizes our strategy and the results obtained with it.)

In initial experiments, we qualitatively compared MT staining patterns with concentrations of the $\mathrm{YL} 1 / 2$ antibody ranging from $1: 200$ to $1: 2,000,000$. At $1: 2000$, the staining was essentially similar to that at $1: 200$ (see above), while at 1:20,000, the staining was only slightly lower in density. At 1:2,000,000, the staining was drastically reduced to only occasional gold particles. At 1:200,000 (which proved to be the most useful antibody concentration for the present studies), both the main shaft and distal region of the axon contained stained polymer, but the levels and/or density of staining were lower than with the 1:200 dilution. These results indicate that the 1:200 dilution that we have routinely used is an extremely high concentration of the antibody for detecting tyrosinated $\alpha$-tubulin in axonal MTs, and 


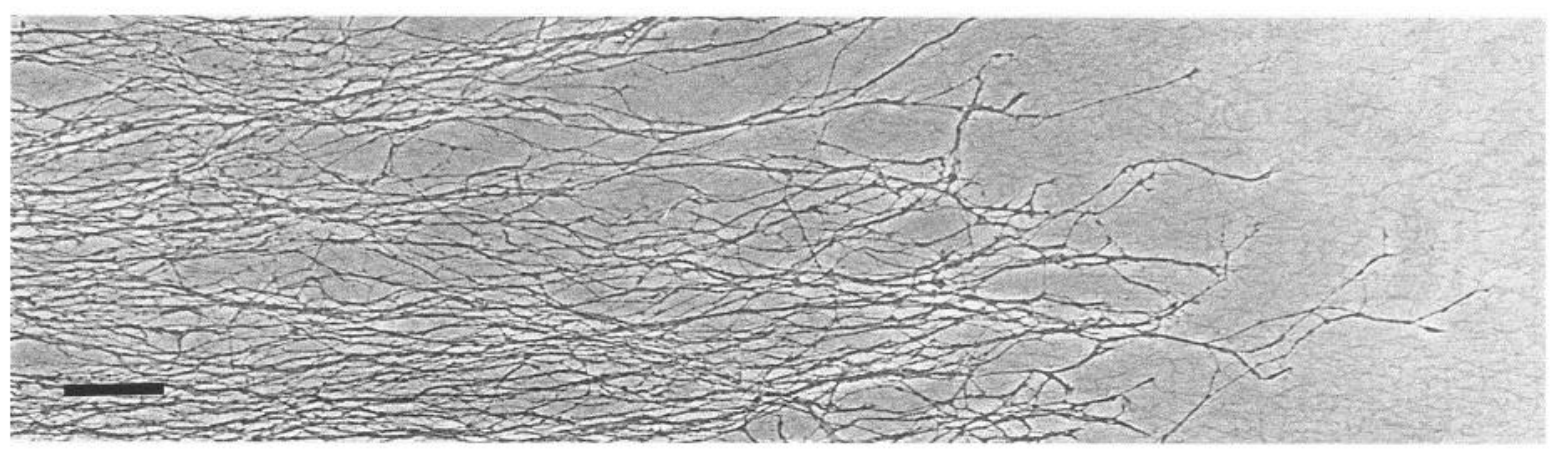

Figure 3. Phase-contrast micrograph showing a portion of an explant culture, the type of culture used for immunoelectron microscopic analyses in the present study. The micrograph shows an area dense in the distal regions of the axons, with a relatively synchronous array of growth cones extending toward the right of the micrograph. The main shafts of the axons are toward the left. More precise estimates of distances from axon tips were obtained at the electron microscope, using the neurofilament array as an unambiguous and nocodazole-resistant marker that typically terminates a few microns proximal to the MT array. Scale bar, $70 \mu \mathrm{m}$.

that the $1: 200,000$ dilution could afford us the opportunity to detect potential differences in tyrosination state that are lost due to "overexposure" at 1:200. For this reason, our quantitative efforts were focused on comparing the results obtained using the $1: 200$ and $1: 200,000$ dilutions of the antibody.

At $1: 200,50 \%$ of the MT polymer in the main shaft stained for tyrosinated $\alpha$-tubulin (Fig. 4a), and almost all of this is labile (see Baas and Black, 1990). Among this stained polymer, the vast majority (91\%) stained densely, with 150-250 gold particles per micron of MT, while the remaining $9 \%$ stained with a somewhat lower density of $40-80$ gold particles per micron of MT. After $3 \mathrm{~min}$ in nocodazole, qualitative examination reveals little difference in the staining pattern (Fig. 4d). However, quantitative analyses revealed a modest increase (to $14 \%$ ) in the proportion of stained polymer with the lower density of gold particles, suggesting that the more densely stained polymer may be preferentially enriched in subsection I of the labile domains. However, it is pertinent that even after $15-30 \mathrm{~min}$ in nocodazole, after which greater than $95 \%$ of the stained polymer has depolymerized, a portion of polymer remaining is of the more densely stained type (Baas and Black, 1990). In addition, the most dramatic transition regions that we have observed between the stable and labile domains were those in which subsection II (the portion of the labile domain continuous with the stable domain) was densely stained (Baas and Black, 1990; Baas and Ahmad, 1992; see also Fig. 5). In both subsections of MTs in the distal axon, all of the polymer stained with the higher density of gold particles (Fig. $4 b, c$ ), as did the polymer remaining in subsection II after 3 min of nocodazole (Fig. 4e), during which all of subsection I depolymerized (Fig. $4 f$ ). Collectively, these results at the 1:200 antibody dilution provide some evidence for variability in tyrosination state among, and perhaps along, individual labile domains, but are not extremely compelling in this regard.

In our previous studies on the main shaft region of the axon, we quantified the proportion of MT profiles in our electron micrographs that showed the transition region between the stable and labile domains (Baas and Black, 1990). The proportion was low, but given the lengths of the profiles relative to the total lengths of the MTs, was consistent with the idea that most or all of the MTs in the axon consist of both domains. Given that the distal axon is enriched in labile domains, and that the average length of a labile domain is $\approx 50 \mu \mathrm{m}$, we reasoned that a higher proportion of the profiles in our electron micrographs might show both domains if we searched for them specifically in the region of the axon roughly $50 \mu \mathrm{m}$ from the axon tip. Consistent with this prediction, we readily found three transition regions during random searches of a small cluster of axons examined in this region (Fig. 5). As in our previous work, all transition regions clearly show a densely stained labile domain situated at the plus end of an unstained stable domain.

Studies performed at 1:200,000, when considered together with the data at 1:200, were more compelling with regard to the issue of regional differences, and more closely reflect the results at the immunofluorescence level. Compared to $1: 200$, the proportion of polymer that stained in the main shaft dropped to only $5 \%$, and the density of staining on this polymer was only 40-80 gold particles per micron of MT (Fig. $6 a$ ). This indicates that the majority of the labile polymer in the main shaft does not stain for tyrosinated $\alpha$-tubulin at this lower antibody concentration. In contrast, the reduction in staining in the distal axon was far less dramatic; $98 \%$ of the polymer in subsection I of the MTs (Fig. $6 b$ ) stained, as did $96 \%$ in subsection II (Fig. $6 c$ ). The polymer in subsection I stained with a density of 40 150 gold particles per micron, while the polymer in subsection II stained with a density of $40-80$ gold particles per micron, both higher than the staining density in the mainshaft. After 3 min in nocodazole, the proportion of stained polymer dropped to $0.5 \%$ in the main shaft (Fig. $6 d$ shows a typical region with no MT polymer stained), but remained high, $95 \%$, in subsection II of the labile domains in the distal axon (Fig. 6e). As expected, all of the polymer in subsection I of the labile domains in the distal axon depolymerized (Fig. $6 f$ ). These results indicate that the portion of the labile domain that depolymerizes most rapidly in the presence of nocodazole is also the portion that contains the highest levels of tyrosinated $\alpha$-tubulin. Thus, higher levels of tyrosinated $\alpha$-tubulin are found in the region of the polymer nearest the plus end of the MT.

Collectively, these findings, summarized in Tables 1 and 2, indicate that there are higher levels of tyrosinated $\alpha$-tubulin in the subsection of the labile domain closer to the plus end of the MT than in the subsection farther from the plus end. These results suggest that there may be a gradient in tyrosination state along the length of the labile domain, with the levels of tyrosinated $\alpha$-tubulin increasing toward the plus end of the MT. These results and speculations apply to the labile domains in both the main shaft and distal regions of the axon. In addition, however, there is a clear difference in the levels of tyrosinated 

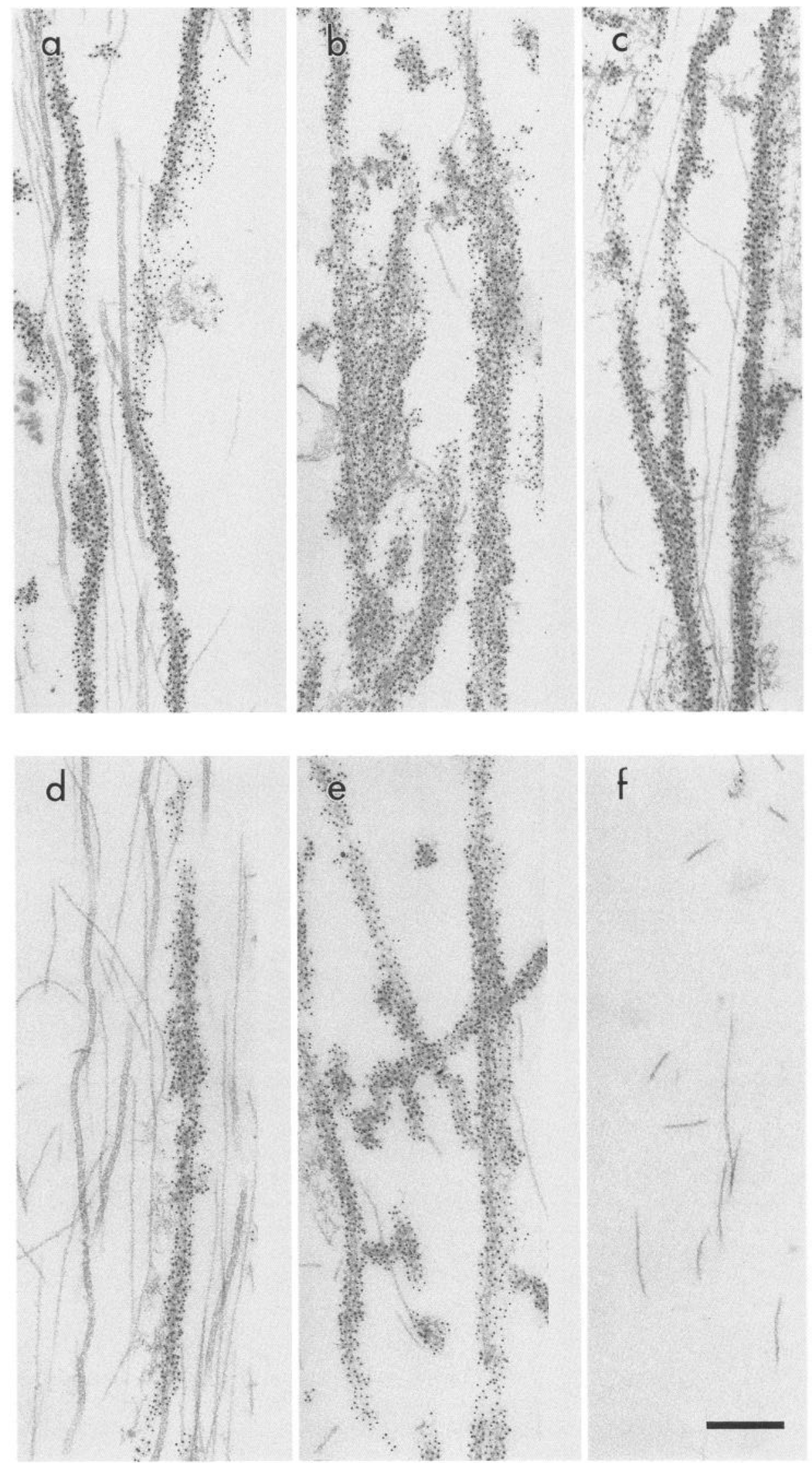

Figure 4. Immunoelectron micrographs of axonal MTs stained for tyrosinated $\alpha$-tubulin with the YL $1 / 2$ antibody used at a concentration of 1:200. $a-c$, respectively, show areas from control axons in the main shaft region, a region roughly $20-40 \mu \mathrm{m}$ from the tip of the axon, and a region roughly $2-10$ $\mu \mathrm{m}$ from the tip. $d-f$, respectively, show comparable regions of axons that were treated for $3 \mathrm{~min}$ with $2 \mu \mathrm{g} / \mathrm{ml}$ nocodazole prior to extraction and preparation for immunoelectron microscopy. In the main shaft, roughly half of the polymer is stained, and this proportion drops only slightly after nocodazole treatment. In both of the distal regions, all of the polymer stained densely. After nocodazole treatment, the region farther from the axon tip is essentially unchanged. In contrast, all of the polymer has depolymerized in the region closer to the axon tip. See Results and Table 1 for more details and quantitative data. Scale bar, $0.25 \mu \mathrm{m}$. 
Table 1. Proportion of microtubule mass that stains for tyrosinated $\alpha$-tubulin at different antibody concentrations

\begin{tabular}{|c|c|c|c|}
\hline & \multirow[b]{2}{*}{ Main shaft } & \multicolumn{2}{|c|}{ Distal region } \\
\hline & & $\begin{array}{l}20-40 \mu \mathrm{m} \\
\text { from tip }\end{array}$ & $\begin{array}{l}2-10 \mu \mathrm{m} \\
\text { from tip }\end{array}$ \\
\hline \multicolumn{4}{|l|}{$1: 200$} \\
\hline Undrugged & $50 \%$ & $100 \%$ & $100 \%$ \\
\hline $3 \mathrm{~min}$ nocodazole & $42 \%$ & $100 \%$ & $-^{*}$ \\
\hline \multicolumn{4}{|l|}{$1: 200,000$} \\
\hline Undrugged & $5 \%$ & $96 \%$ & $98 \%$ \\
\hline $3 \mathrm{~min}$ nocodazole & $0.5 \%$ & $95 \%$ & $-^{*}$ \\
\hline
\end{tabular}

Polymer was scored as stained or unstained, and the lengths of stained and unstained polymer were scored and summed separately. Percentages of total polymer stained are listed for each axon region, antibody concentration, and drug treatment as described in the text. For each category in both Table 1 and Table 2, a total of at least $500 \mu \mathrm{m}$ of polymer were analyzed. As indicated in the text, our goals were two-fold. First, we wished to compare the levels of tyrosinated $\alpha$-tubulin in subsection I (closer to the axon tip) and subsection II (farther from the axon tip) of the labile domains of MTs terminating in each of the two regions of the axon, the main shaft and distal regions. Second, we wished to compare the levels of tyrosinated $\alpha$-tubulin in the labile domains of MTs terminating in each of these two axon regions relative to one another. Distinguishing the subsections of the labile domains in the distal axon was relatively simple, as subsection II corresponds to a region $20-40 \mu \mathrm{m}$ from the axon tip, and subsection I corresponds to a region $2-10 \mu \mathrm{m}$ from the axon tip. This was not possible in the main shaft because individual MTs are staggered along its length (see Fig. 1). Thus, the subsections were pharmacologically dissected apart using nocodazole (see text for more details).

* No polymer remaining after drug treatment.

$\alpha$-tubulin in the labile domains in these regions. While tyrosinated polymer is present in both the main shaft and distal axon, the polymer in the distal axon contains higher levels of tyrosinated $\alpha$-tubulin than does the polymer in the main shaft. These results indicate that the polymer in the distal region of the axon is particularly dynamic.

\section{Discussion}

In the present study, we have used tyrosination state as an indirect indicator of MT polymer age to compare the dynamic properties of the MT polymer extending into the distal region of the axon with the properties of the MT polymer terminating in the main shaft. Previous observations indicated that the MT polymer in the former region is collectively more dynamic than
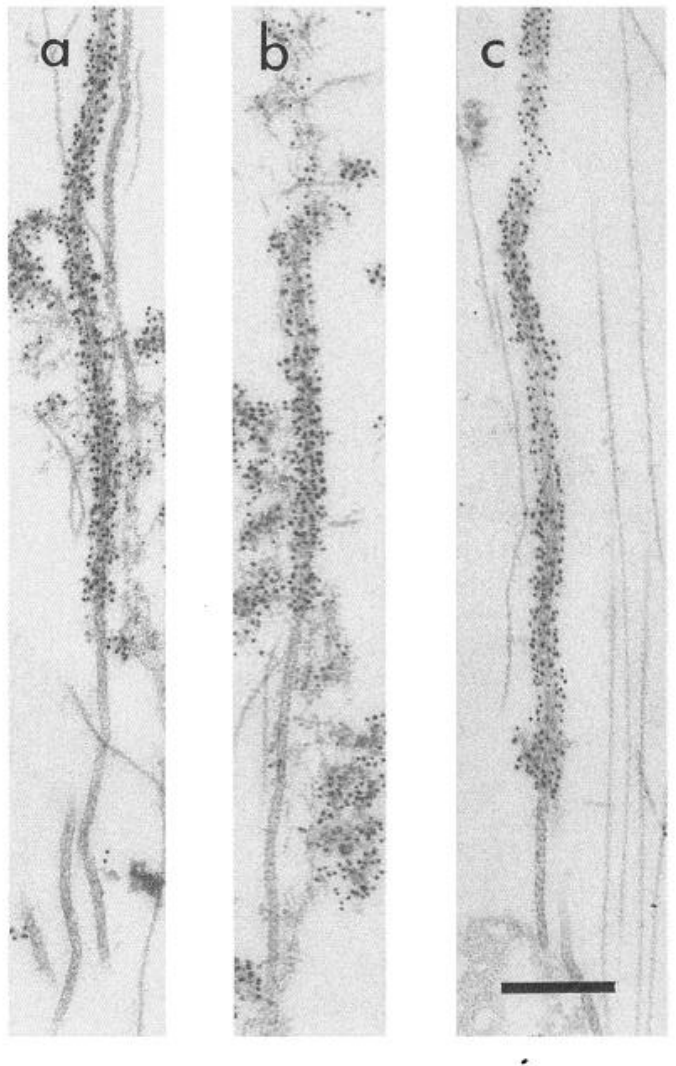

Figure 5. Immunoelectron micrographs of three different MT profiles showing the transition region between the stable and labile domains. The labile domain is densely stained for tyrosinated $\alpha$-tubulin, while the stable domain is unstained. All three profiles were found during a random search of a small cluster of axons viewed roughly $50 \mu \mathrm{m}$ from the axon tip, a region expected to contain an unusually high number of transition regions (see text and Fig. 1). Plus ends of MTs are directed upward in the panels. Scale bar, $0.25 \mu \mathrm{m}$.

the MT polymer in the latter region (see introductory remarks). However, these studies relied on techniques of insufficient resolution to compare the dynamics of individual MTs within the array, and thus do not distinguish between two possible explanations. The first possibility is that the polymer extending into the distal axon is no more dynamic than the labile polymer

Table 2. Density of staining for tyrosinated $\alpha$-tubulin as measured in gold particles per micron of microtubule polymer

\begin{tabular}{|c|c|c|c|}
\hline & \multirow[b]{2}{*}{ Main shaft } & \multicolumn{2}{|l|}{ Distal region } \\
\hline & & $20-40 \mu \mathrm{m}$ from tip & $2-10 \mu \mathrm{m}$ from tip \\
\hline \multicolumn{4}{|l|}{$1: 200$} \\
\hline Undrugged & $\begin{array}{c}150-250(91 \%) \\
40-80(9 \%)\end{array}$ & $150-250(100 \%)$ & $150-250(100 \%)$ \\
\hline 3 min nocodazole & $\begin{array}{c}150-250(86 \%) \\
40-80 \quad(14 \%)\end{array}$ & $150-250(100 \%)$ & $-^{*}$ \\
\hline \multicolumn{4}{|l|}{$1: 200,000$} \\
\hline Undrugged & $40-80 \quad(100 \%)$ & $40-80 \quad(100 \%)$ & $40-150(100 \%)$ \\
\hline 3 min nocodazole & $40-80 \quad(100 \%)$ & $40-80 \quad(100 \%)$ & $-^{*}$ \\
\hline
\end{tabular}

That fraction of the total polymer that was stained was further analyzed in terms of the density of gold per micron of MT polymer. Densities are listed as ranges. Under some conditions, two different densities were clearly apparent, and these were scored separately as indicated. Percentages of the stained polymer in each density range are provided in parentheses. Under the other conditions, there was some variability, but multiple classes were not apparent, and hence are listed as a single range.

* No polymer remaining after drug treatment. 

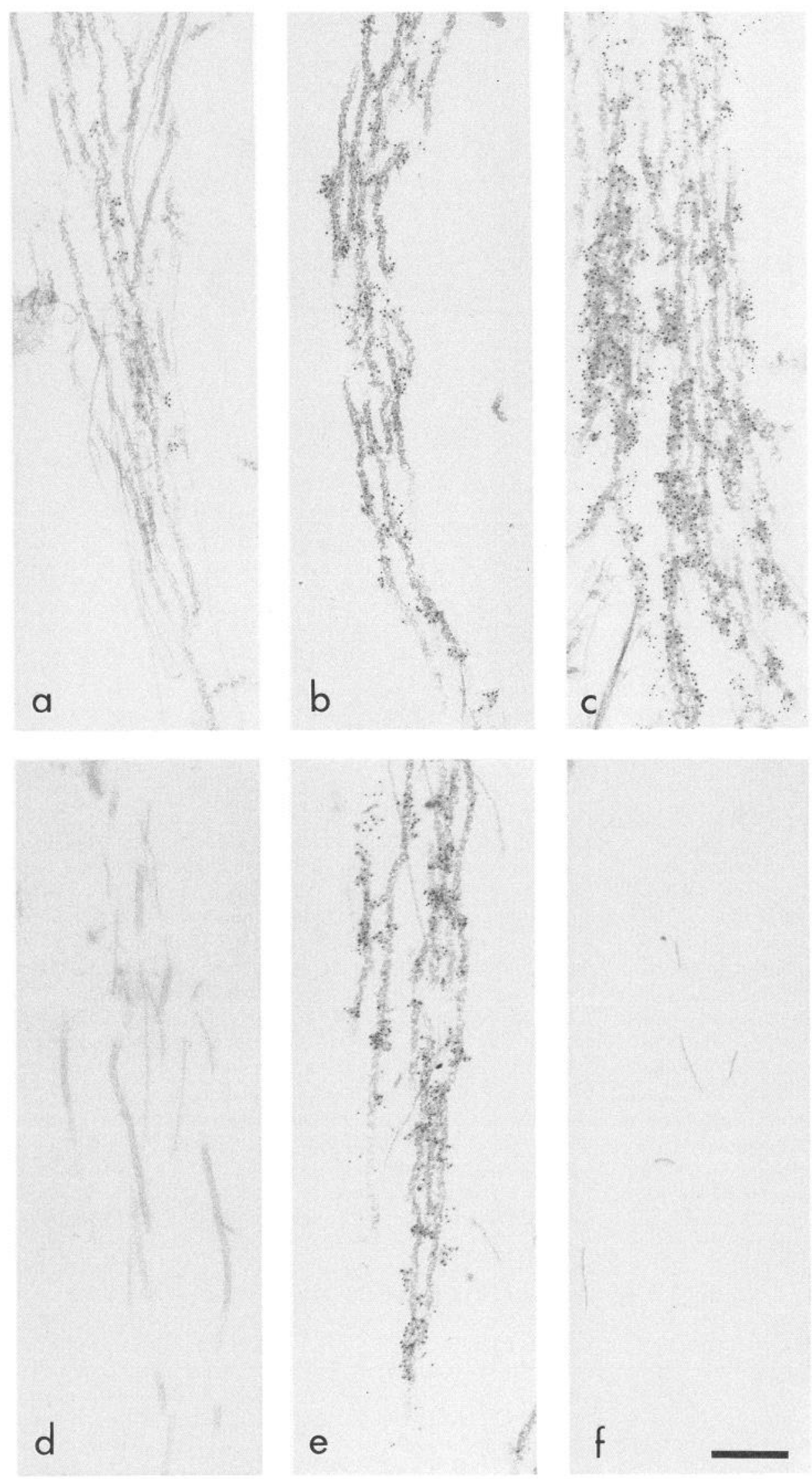

Figure 6. Immunoelectron micrographs of axonal MTs stained for tyrosinated $\alpha$-tubulin with the YL $1 / 2$ antibody used at a concentration of $1: 200,000 . a-c$, respectively, show areas from control axons in the main shaft region, a region roughly $20-40 \mu \mathrm{m}$ from the tip of the axon, and a region roughly $2-10 \mu \mathrm{m}$ from the tip. $d-f$, respectively, show comparable regions of axons that were treated for $3 \mathrm{~min}$ with $2 \mu \mathrm{g} / \mathrm{ml}$ nocodazole prior to extraction and preparation for immunoelectron microscopy. Quantitative analyses indicate that in the main shaft, $\approx 5 \%$ of the polymer is stained, and the rest is unstained. In the distal regions farther from and closer to the axon tip, respectively, $\approx 96 \%$ and $\approx 98 \%$ of the polymer is stained. The density of staining is higher in the most distal region compared to the main shaft and penultimate region. After nocodazole treatment, a significantly lower proportion of the polymer in the main shaft is stained $(0.5 \%$; not shown), the distal region farther from the axon tip is essentially unchanged, and all of the polymer has depolymerized from the region nearer the axon tip. See Results and Table 1 for more details. Scale bar, $0.25 \mu \mathrm{m}$. 


\section{STABLE DOMAIN LABILE DOMAIN}

\section{MAINSHAFT} MICROTUBULE
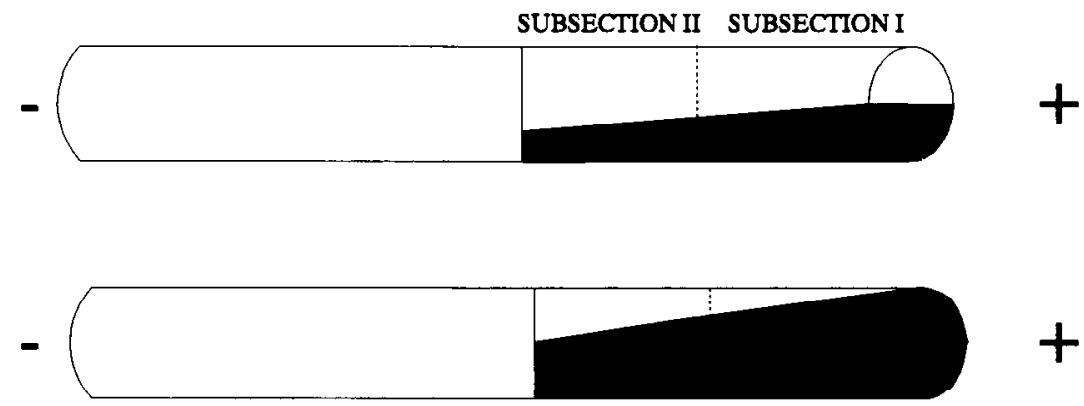

\author{
DISTAL \\ MICROTUBULE
}

\section{LEVELS OF TYROSINATED ALPHA TUBULIN}

Figure 7. Schematic illustrating our proposal for the distribution of tyrosinated $\alpha$-tubulin along the length of individual MTs in the main shaft and distal regions of the axon. Each MT consists of a labile domain situated at the plus end of a stable domain. Because of the great length of axonal MTs $(\approx 100 \mu \mathrm{m})$, the fact that the stable and labile domains each generally comprise roughly half of the total length of the MT, and because of their plus-end-distal polarity orientation, stable domains are confined to the main shaft region of the axon, while labile domains are present throughout the axon. To investigate whether the levels of tyrosinated $\alpha$-tubulin vary along the length of the labile domain, we arbitrarily divided the labile domains into two subsections termed I and II. Because subsection I is situated at the plus end of the MT, it will depolymerize in the presence of nocodazole before subsection II. We have discovered that $3 \mathrm{~min}$ in $2 \mu \mathrm{m}$ nocodazole is sufficient to depolymerize subsection I while leaving most of subsection II intact. Using length measurements to visualize separately subsections I and II of the MTs in the distal axon, and 3 min nocodazole treatments to dissect apart subsections I and II in the main shaft (and distal) regions of the axon, we were able to compare the $\alpha$-tubulin levels in both subsections of the labile domains of MTs in both axon regions (see Tables 1 and 2 ). In both regions of the axon, the stable domains of the MTs contain extremely little or no tyrosinated $\alpha$-tubulin. In contrast, the labile domains contain significantly higher levels of tyrosinated $\alpha$-tubulin. Based on our data on the levels of tyrosinated $\alpha$-tubulin in the two subsections of the labile domain, we propose that the levels progressively increase as one moves from the stable domain toward the plus end of the MT. In addition, and most dramatically, it is clear that the overall levels of tyrosinated $\alpha$-tubulin are higher in the labile domains in the distal region of the axon compared to the labile domains in the main shaft.

situated at the plus ends of MTs staggered all along the length of the axon. The second possibility is that the individual labile domains extending into the distal axon are more dynamic than the individual labile domains terminating in the main shaft. Our studies, using the high degree of resolution afforded by immunoelectron microscopy, provide compelling evidence in favor of this latter possibility. Based on our data, we propose that there is a gradient of increasing levels of tyrosinated $\alpha$-tubulin in the labile domain moving from the stable domain toward the plus end of the MT, and that this applies to MTs in both the main shaft and distal regions of the axon (Fig. 7 is a schematic summary). From these observations, it follows that the labile domains throughout the axon undergo rapid bouts of assembly/disassembly, and that these bouts sometimes involve the entire labile domain, but at other times involve only a portion of it. As a result, higher levels of tyrosinated $\alpha$-tubulin are present closer to the plus end of the MT, the site from which assembly and disassembly occur (Baas and Ahmad, 1992). In addition, the levels of tyrosinated $\alpha$-tubulin in the labile domains extending into the distal axon are clearly higher than those in the labile domains terminating in the main shaft region of the axon. These considerations indicate that, while highly dynamic MT polymer exists throughout the axon, the polymer extending into the distal axon is particularly dynamic.

In the past, observations suggesting that the MT polymer in the distal region of the axon may be especially dynamic have been used to argue that the net addition of new polymer to the growing axon occurs in this distal region, behind the advancing growth cone (Bamburg et al., 1986; Mitchison and Kirschner,
1988; Robson and Burgoyne, 1988; Lim et al., 1989, 1990). Although our data are consistent with the observations upon which this argument is based, there is a growing body of evidence suggesting that the argument may be otherwise flawed. For example, it is still unclear how local MT dynamics are related to length changes of axonal MTs, or whether local dynamics result in any net increase in total polymer anywhere in the axon. For example, it is possible that MT assembly and disassembly events are perfectly balanced for each MT. If this is correct, then the total length of the MT will remain the same, and no net addition of polymer will occur as a result of local dynamics. Alternatively, assembly and disassembly events may be perfectly balanced within the MT array, but not for individual MTs. If this is correct, then the lengths of individual MTs will change, but again, the total polymer mass in the axon will remain the same. If the total polymer mass is not increased by local dynamics, then other mechanisms are clearly necessary to elevate the polymer levels during axon growth. At present, the possibility that such alternate mechanisms exist is no less viable than the idea that the degree of MT assembly in the axon exceeds the degree of disassembly.

In fact, the idea that the MT array of the axon is expanded simply by the distal addition of subunits is problematic in that it fails to explain how sufficient levels of tubulin subunits could be moved from their site of synthesis in the cell body to the distal region of the axon. Although for very short axons, diffusion would be sufficient to carry the tubulin to the growth conc, this is clcarly not the casc once that axon has cxcccded a length of roughly a few hundred microns. Thus, at present, it 
seems wisest to harken back to an earlier model, which held that MT polymer is preassembled in the cell body and actively transported down the axon as the assembled polymer (Lasek, 1982, 1988). During transit, the subunits of different MTs shuffle among themselves, resulting in a shift during transit from large numbers of short MTs to smaller numbers of longer MTs. This model incorporates local dynamics as a key principal, but also satisfactorily explains how sufficient levels of tubulin for axon growth could be translocated over distances too great to be explained simply by diffusion. In addition, this model is consistent with the huge body of kinetic data derived from axonal transport work (Black and Lasek, 1980; for review, see Lasek, 1988), and recent studies using real-time imaging to observe the simultaneous length changes and movements of fluorescently labeled MTs in 2 different types of axons (Reinsch et al., 1991; Sabry et al., 1991; Tanaka and Kirschner, 1991). Finally, this model is consistent with the notion that the distal region of the axon could be a major site of MT assembly, because of the enrichment of MT plus ends in this region. However, with regard to this latter point, it is pertinent that recent work from our laboratory indicates that very little of the highly dynamic polymer in the distal axon is ever converted into stable polymer, whereas much of the dynamic polymer in the proximal axon is ultimately stabilized (P. W. Baas, F. J. Ahmad, T. P. Pienkowski, A. Brown, and M. M. Black, unpublished data). These observations suggest that the net addition of long-lived MT polymer to the growing axon occurs proximally, not distally.

In light of these considerations, attention shifts to other potential reasons for the presence of particularly dynamic MT polymer in the distal region of the axon. Some insight is provided by recent observations on the behavior of the growth cone at the tip of the distal axon and the cytoskeletal elements within it during axon growth (Goldberg and Burmeister, 1986; Aletta and Greene, 1988; Forscher and Smith, 1988; Sabry et al., 1991; Tanaka and Kirschner, 1991; Rivas et al., 1992). These studies have shown that the growth cone is a very dynamic structure unto itself, and raise the intriguing possibility that the especially dynamic nature of the distal polymer may be linked to the unique mechanical properties of the growth cone. As the axon elongates, the growth cone reacts to its environment with rapid bouts of extensions and retractions. Clearly the internal structures within the axon must react to these events. It is now well established that MTs "invade" the growth cone during its forward movements (Forscher and Smith, 1988; Rivas et al., 1992). This in vasion was originally thought to result from net assembly onto the ends of the MTs, but more recently has been shown to result from a combination of dynamic events and polymer movements (Sabry et al., 1991; Tanaka and Kirschner, 1991). Thus, it is possible that the distal polymer undergoes especially frequent dynamic events not because it is intrinsically any different from the labile polymer in the main shaft, but rather because it is reacting to different environmental conditions. In this view, the particularly dynamic properties of the distal polymer may be directly related to the morphologic plasticity required in the region of the growth cone for pioneering, steering, pathfinding, and consolidation events essential for normal axon growth.

At present, it is not clear how the activities of the distal polymer are coordinated with the behavior of the growth cone. However, available evidence appears to point to some functional connection between the MTs in the distal axon and the actin meshwork of the growth cone. Most notably, it has been shown that dissolution of the actin-rich filopodial region of the growth cone permits the MTs to invade farther into the cone (Forscher and Smith, 1988). This suggests that the actin meshwork may normally act as a barrier to inhibit or regulate the forward movement of the MTs. This barrier may be simply the physical presence of the actin, or may result from forces exerted on the MT array by the actin (Dennerll et al., 1988). Regardless of the precise mechanism, the interplay between the distal MTs and the actin meshwork of the growth cone provides an intriguing example of how the MT polymer in the distal region of the axon confronts different regulatory pressures than does the labile polymer located in the main shaft of the axon. Additional studies will be required to elucidate further how these and other factors interface with one another during the growth of the axon.

\section{References}

Aletta JM, Greene LA (1988) Growth cone configuration and advance: a time-lapse study using video-enhanced differential interference contrast microscopy. J Neurosci 8:1425-1435.

Baas PW, Ahmad FJ (1992) The plus ends of stable microtubules are the exclusive nucleating structures for microtubules in the axon. J Cell Biol 116:1231-1241.

Baas PW, Black MM (1990) Individual microtubules in the axon consist of domains that differ in both composition and stability. $\mathrm{J}$ Cell Biol 111:495-509.

Baas PW, White LA, Heidemann SR (1987) Microtubule polarity reversal accompanies regrowth of amputated neurites. Proc Natl Acad Sci USA 84:5272-5276.

Baas PW, Deitch JS, Black MM, Banker GA (1988) Polarity orientation of microtubules in hippocampal neurons: uniformity in the axon and nonuniformity in the dendrite. Proc Natl Acad Sci USA 85:8335-8339.

Baas PW, Black MM, Banker GA (1989) Changes in microtubule polarity orientation during the development of hippocampal neurons in culture. J Cell Biol 109:3085-3094.

Baas PW, Slaughter T, Brown A, Black MM (1991) Microtubule dynamics in axons and dendrites. J Neurosci Res 30:134-153.

Bamburg JR, Bray D, Chapman K (1986) Assembly of microtubules at the tip of growing axons. Nature 321:788-790.

Black MM, Baas PW (1989) The basis of polarity in neurons. Trends Neurosci 12:211-214.

Black MM, Lasek RJ (1980) Slow components of axonal transport: two cytoskeletal networks. J Cell Biol 86:616-623.

Black MM, Baas PW, Humphries S (1989) Dynamics of $\alpha$-tubulin deacetylation in intact neurons. J Neurosci 9:358-368.

Bray D, Bunge MB (1981) Serial analysis of microtubules of cultured rat sensory neurons. J Neurocytol 10:589-605.

Brown A, Slaughter TS, Black MM (in press) Newly assembled microtubules are abundant in the proximal and distal regions of growing axons. J Cell Biol, in press.

Bulinksi JC, Richards JE, Piperno G (1988) Posttranslational modifications of $\alpha$-tubulin: detyrosination and acetylation differentiate populations of interphase microtubules in cultured cells. J Cell Biol 106:1213-1220.

Dennerll TJ, Joshi HC, Steele VL, Buxbaum RE, Heidemann SR (1988) Tension and compression in the cytoskeleton of $\mathrm{PC}-12$ neurites. II: Quantitative measurements. J Cell Biol 107:665-674.

Dustin P (1984) Microtubules, 2d ed. New York: Springer.

Forscher P, Smith SJ (1988) Actions of cytochalasins on the organization of actin filaments and microtubules in a neuronal growth cone. J Cell Biol 107:1505-1516.

Goldberg DJ, Burmeister DW (1986) Stages in axon formation: observations of growth of Aplysia axons in culture using video-enhanced contrast-differential interference contrast microscopy. J Cell Biol 103: 1921-1931.

Gunderson GG, Khawaja S, Bulinski JC (1987) Postpolymerization detyrosination of $\alpha$-tubulin: a mechanism for subcellular differentiation of microtubules. J Cell Biol 105:251-264.

Heidemann SR, Landers JM, Hamborg MA (1981) Polarity orientation of axonal microtubules. J Cell Biol 91:661-665.

Higgins D, Lein PJ, Osterhout DJ, Johnson MI (1991) Tissue culture 
of mammalian autonomic neurons. In: Culturing nerve cells (Banker G, Goslin K, eds), pp 177-205. Cambridge, MA: MIT Press.

Kilmartin JV, Wright B, Milstein C (1982) Rat monoclonal antitubulin antibodies derived by using a new nonsecreting rat cell line. J Cell Biol 93:576-582.

Kirschner M, Mitchison T (1986) Beyond self-assembly: from microtubules to morphogenesis. Cell 45:329-342.

Lasek RJ (1982) Translocation of the neuronal cytoskeleton and axonal locomotion. Philos Trans R Soc Lond [Biol] 299:313-327.

Lasek RJ (1988) Studying the intrinsic determinants of neuronal form and function. In: Intrinsic determinants of neuronal form and function (Lasek RJ, Black MM, eds), pp 1-58. New York: Liss.

Lim SS, Sammak PJ, Borisy GG (1989) Progressive and spatially differentiated stability of microtubules in developing neuronal cells. J Cell Biol 109:253-264.

Lim SS, Edson KJ, Letourneau PC, Borisy GG (1990) A test of microtubule translocation during neurite elongation. J Cell Biol 111: 123-130.

Meininger V, Binet S (1989) Characteristics of microtubules at the different stages of neuronal differentiation and maturation. Int Rev Cytol 114:21-79.

Mitchison T, Kirschner M (1988) Cytoskeletal dynamics and nerve growth. Neuron 1:761-772.

Okabe S, Hirokawa N (1988) Microtubule dynamics in nerve cells; analysis using microinjection of biotinylated tubulin into $\mathrm{PC} 12$ cells. J Cell Biol 107:651-664.

Piperno G, Fuller MT (1985) Monoclonal antibodies specific for an acetylated form of $\alpha$-tubulin recognize antigens in cilia and flagella from a variety of organisms. J Cell Biol 101:2085-2094.
Reinsch SS, Mitchison TJ, Kirschner M (1991) Microtubule polymer assembly and transport during axonal elongation. J Cell Biol 115: 365-379.

Rivas RJ, Burmeister DW, Goldberg DJ (1992) Rapid effects of laminin on the growth cone. Neuron 8:107-115.

Robson SJ, Burgoyne RD (1988) Differential levels of tyrosinated, detyrosinated, and acetylated alpha-tubulins in neurites and growth cones of dorsal-root ganglion neurons. Cell Motil Cytoskel 12:273282.

Sabry JH, O'Connor TP, Evans L, Toroian-Raymond A, Kirschner MW, Bentley D (1991) Microtubule behavior during guidance of pioneer neuron growth cones in situ. J Cell Biol 115:381-395.

Sherwin T, Gull K (1989) Visualization of detyrosination along single microtubules reveals novel mechanisms of assembly during cytoskeletal duplication in trypanosomes. Cell 57:211-221.

Tanaka EM, Kirschner MW (1991) Microtubule behavior in the growth cones of living neurons during axon elongation. J Cell Biol 115:345363.

Tsukita S, Ishikawa H (1981) The cytoskeleton in mylinated axons: serial section study. Biomed Res 2:424-437.

Wheland J, Willingham MC, Sandoval IV (1983) A rat monoclonal antibody reacting specifically with the tyrosinated form of $\alpha$-tubulin. Biochemical characterization. Effects on microtubule polymerization in vitro and microtubule polymerization and organization in vivo. J Cell Biol 97:1476-1490.

Whitlon DS, Baas PW (1992) Improved methods for the use of glass coverslips in cell culture and electron microscopy. $\mathrm{J}$ Histochem $\mathrm{Cy}-$ tochem $40: 875-877$. 